\title{
Perfecting evaluation methods of energy equipment technical condition and resource based on electromagnetic-acoustic effect
}

\author{
$M G$ Bashirov*, $N N$ Luneva, $I G$ Khusnutdinova, $E M$ Bashirova, $N M$ Zakharov and $R R$ Gaziev \\ A subdivision of FSBEO HE Ufa State Oil Technical University in Salavat
}

\begin{abstract}
To assess the technical condition and resource for the safe operation of electric and electrical equipment, it is proposed to use the electromagnetic-acoustic effect and the dynamic identification method based on the analysis of the coordinates of the roots of the characteristic equation (polynomial of the denominator of the transfer function of the system "control object - electromagnetic-acoustic transducer") on the complex plane. The transfer function of the studied system "control object - electromagneticacoustic transducer" is an integral quantitative parameter characterizing the state of the equipment metal.
\end{abstract}

\section{Introduction}

Failures of electric power and electrical equipment can cause disruptions of power supply and the continuity of technological processes, are accompanied by significant economic and environmental damage, pose a threat to the life of personnel of industrial enterprises and general population [1]. According to official statistics over the past thirty years, in the main buildings of thermal power plants (TPPs), about 30 major accidents occurred with the failure of more than one unit, with about $90 \%$ of major accidents caused by equipment failures and accompanied by fire, and $10 \%$ being a consequence of damage to building structures. The share of accidents that occurred in engine rooms is $72 \%$ of the total, in boiler rooms - $23 \%$ and in cable tunnels - about $5 \%$ [2]. The main power equipment of thermal power plants, the most exposed to mechanical stress, is a turbogenerator. Long-term operation under intense conditions of exposure to cyclic loads leads to damage due to metal fatigue [3]. As a result, defects occur in the metal structural elements of the generator, leading to the generator failure.

Among the defects, the presence and degree of development of which are the key factor in determining the reliability of operation and the physical life of the turbogenerator, we should single out the rotor defects. The most dangerous rotor shaft defect is fatigue cracks. The main prerequisite for their appearance is the formation of local stress concentrators, which makes particularly important the issue of controlling the stressstrain state and damage of structures in the process of their entire life cycle. Already at the stages of manufacture and installation in the equipment, fields of technological stresses and deformations are formed, which contribute to the accumulation of damage in the metal structure. For the timely detection of areas of metal structures that are most prone to damage, it is necessary to know their actual stress-strain state [4 - 7].

To detect defects in the metal of electric power equipment, various non-destructive testing methods are used; there is no universal method capable of detecting the most diverse defects in nature. The main disadvantage of the non-destructive testing methods currently used to assess the state of metal in power equipment is their focus on the search for developed defects, their inability to carry out early diagnostics [8].

In recent years, considerable attention has been paid to the development and application of non-destructive methods for assessing the technical condition of products based on the electromagnetic-acoustic (EMA) effect, which allows generating acoustic waves in the target object, thus receiving information about its technical condition [9 - 11]. Although today only certain parameters of acoustic waves are used, for example, a change in the time of their propagation, amplitude, phase, and the difference in the propagation time of transverse and longitudinal waves. The informative potential of the EMA effect is not fully realized, for example, in metal objects, the parameters of the EMA signal are structurally sensitive, since they clearly respond to the evolution of their structure and stressstrain state. As an integral parameter characterizing the combination of mechanical, acoustic, magnetic, and electrical properties of a metal, one can use the transfer function of the test object. It is a mathematical model of the dynamics of changes in the indicated properties of the object and represents the ratio of the increments of the response signal of the object and the signal of external influence recorded in the operator form under zero initial conditions [12].

Changes in the structure of the metal, the nucleation and development of damage are reflected in the values of the parameters of the transfer function - the coefficients of the polynomials of the numerator and denominator

*Corresponding author: eapp@yandex.ru 


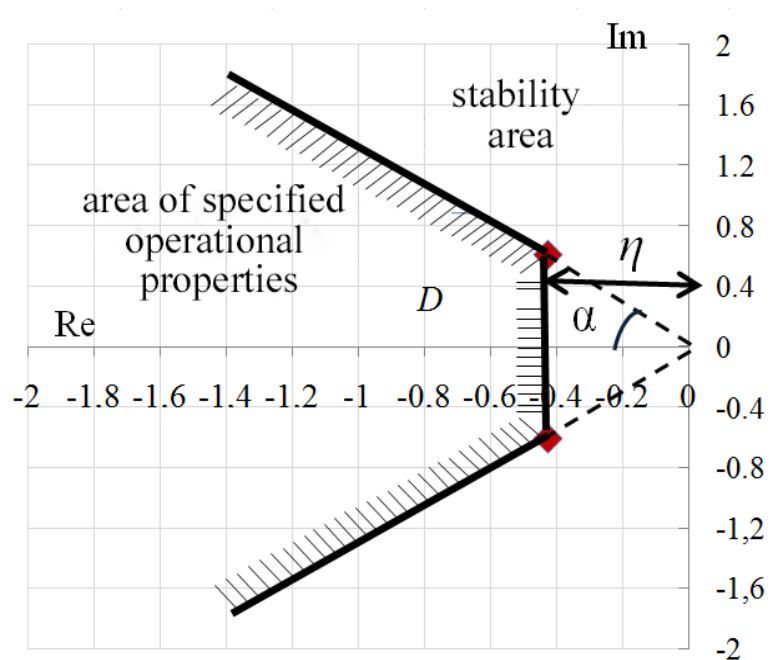

a)

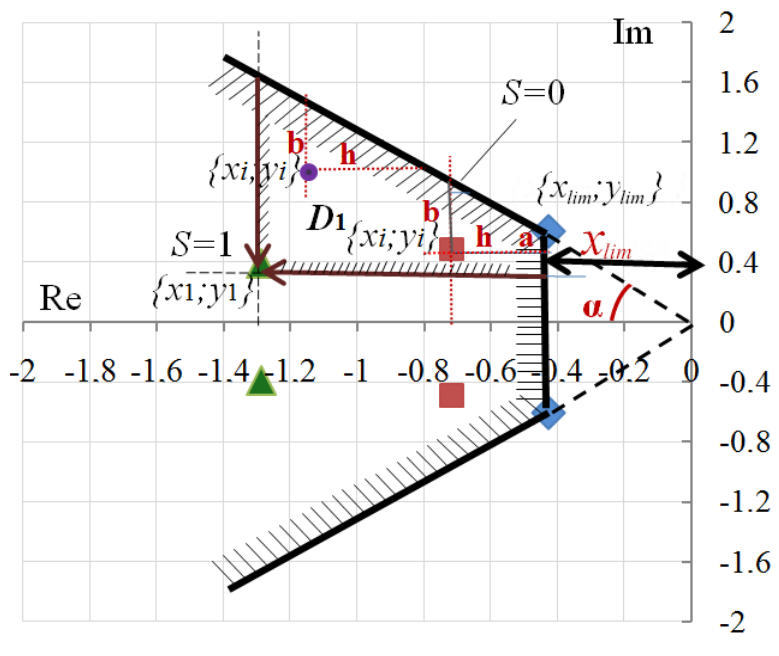

b)

Fig. 1. Assessment of the current technical condition and resource of the pipeline according to the coordinates of the roots of the characteristic polynomial ( $\mathrm{a}$ is the area of the specified operational properties of the pipeline $\mathrm{D}$; $\mathrm{b}$ is the area of operational state of the pipeline $\left.\mathrm{D}_{1}\right)$.

and, accordingly, the values of the roots of polynomials. Therefore, the analysis of the parameters of the transfer function allows us to identify these changes [13].

In general, the transfer function of any system in operator form can be written as.

$$
W(p)=\frac{Y(p)}{X(p)}=K \frac{b_{0}+b_{1} \cdot p+\ldots+b_{m} \cdot p^{m}}{a_{0}+a_{1} \cdot p+\ldots+a_{n} \cdot p^{n}}=K \frac{B(p)}{A(p)},
$$

where $Y(p)$ and $X(p)$ are the increments of the response signals and external influences; $B(p)$ and $A(p)$ are polynomials of the numerator and denominator; $K$ is the gain; $a_{i}, b_{i}$ are the coefficients of polynomials.

In contrast to the classical understanding of the transfer function of an object as a mathematical model with constant parameters, we considered the transfer function as the integral characteristic described above [14].

By tracking the migration of the coordinates of the roots of the characteristic polynomial on the complex plane, we can evaluate the change in the properties of the control object, on the basis of which the limit state of the metal is determined and the remaining lifetime of the equipment is estimated. For an integral quantitative assessment of the state of the control object, the area of the complex plane region is used, beyond which the values of the roots of the characteristic polynomial for given operational properties of the object should not go. The coordinates of the roots, which are the closest imaginary values $\mathrm{x}_{\lim }$ and the most remote from the axis real values $y_{\text {lim, }}$ determine when the object reaches its ultimate state, outline the boundaries of the area of the specified operational properties of the object D (Figure 1). The coordinates of the roots in the initial (healthy) state of the object $\left\{\mathrm{x}_{1}, \mathrm{y}_{1}\right\}$ together with the boundaries of the region of the specified operational properties D determine the boundaries of the region of the healthy state of the object $D_{1}$, which characterizes the remoteness of the initial state of the object from its limiting state.

Quantitatively, $\mathrm{D}_{1}$ can be expressed by the formula

$$
D_{1}=\int_{x_{\text {lim }}}^{x_{1}}\left(\operatorname{tg} \alpha \cdot x-y_{1}\right) d x
$$

where $\operatorname{tg} \alpha=\mathrm{y}_{\text {lim }} / \mathrm{x}_{\text {lim }}$.

During the operation of the object or during its testing, the coordinates of the root characteristic polynomial $\left\{\mathrm{x}_{\mathrm{i}}, \mathrm{y}_{\mathrm{i}}\right\}$ migrate to the boundaries of the region of the specified operational properties $\mathrm{D}$, and the region of the operable state of the object decreases from the initial value $D_{1}$ to zero when the limit state is reached. This current healthy state area was designated $\mathrm{D}_{\mathrm{i}}$,

$$
D_{i}=\int_{x_{\text {lim }}}^{x_{1}}\left(\operatorname{tg} \alpha \cdot x-y_{i}\right) d x
$$

The ratio $S=D_{\mathrm{i}} / D_{1}$, which characterizes the technical condition and remaining lifetime of the object, was called an integral safety parameter. As the resource of an object is exhausted, its value decreases from 1 to 0 $[15,16]$.

Changes in the operation of electric power and electrotechnical equipment of the electrical, magnetic and acoustic properties of the metal directly affect the values of the time and frequency parameters of the EMA signal. This significantly expands the informativeness of the EMA effect in comparison with the traditional use of only acoustic wave parameters, when only the acoustic properties of the metal are analyzed. The use of the transfer function - a mathematical model of the dynamics of changes in the electrical, magnetic, and 
acoustic properties of a metal - as a tool for assessing the technical condition and predicting a remaining lifetime, makes it possible to increase the operational reliability and safety of electric power and electrical equipment.

\section{References}

1. S.A. Belyaev, V.V. Litvak, S.S. Solod, Reliability of TPP thermal power equipment: study allowance, 218 (2008)

2. V.V. Belov, B.K. Pergamenchik, Major accidents at the TPP and their impact on the layout of the main buildings, Engineering exploration and survey of buildings. Special construction. The Herald of the Issu, 4, 61-69 (2013)

3. Defects of powerful turbo generators and methods of their detection of [Digital resource]. Access mode: http://elektro-nt.ru/turbogeneratorsdiagnostic/survey-methods.html.

4. V.F. Rezynskiy, Contemporary problems related to the provision of reliable and safe operation of thermal power plant equipment, Reliability and safety of energy, 1 (2008)

5. M.S. Saikova, Effect of technological defects on the strength and resource of metal hulls of thermal mechanical equipment TPP at thermomechanization impacts: abstract thesis of the Ph.D. thesis (2011)

6. Causes and consequences of accidents and failures of turbo generators. Access mode: http://elektront.ru/investigation-of-the-causes-ofaccidents/accidents-faults-of-turbogenerators/15causes-consequences-of-accidents-and-failures-ofturbine-generators.html.

7. Reliability of turbo generators. Maintaining the operational reliability of the [Digital resource]. Access mode: http://pue8.ru/elektricheskiemashiny/289-nadezhnost-turbogeneratorovpodderzhanie-ekspluatatsionnoj-nadezhnosti.html.

8. O.S. Holodnova, Typical defects of turbo generators and the possibility of pre-empting failures by diagnosing [Digital resource]: http://ensafe.ru/index.php?option=com_content\&vi ew $=$ article \&id $=21 \&$ Itemid $=26$.

9. G.A. Alers, Electromagnetic induction of ultrasonic waves: EMAT, EMUS, EMAR, 16th World Conference on NDT (2004) [Digital resource]: http://www.ndt.net/article/wcndt2004/pdf/noncontact ultrasonics/691_alers.pdf.

10. L. Borja, Weld inspection with EMAT using guided waves, The e-Journal of NDT (2008) [Digital resource]:http://www.ndt.net/article/v13n06/lopez.p df.

11. D. Cziraki, A. Danis, Cost-effective pipeline girth weld AUT for Brazil, Insight, 49(3), 130-131 (2007)

12. I.G. Khusnutdinova, M.G. Bashirov, The use of electromagnetic-acoustic method for estimat-ing the stress-strain state of the metallic elements of power
equipment,Key Engineering Materials, 743, 463467 (2017)

13. M.G. Bashirov, The dynamic identification of the technical condition of pipe-lines on the basis of the analysis of the temporal characteristics of electromagneti-cacoustic signal, IOP Confer-ence Series: Materials Science and Engineering, 511 (2011)

14. M.G. Bashirov, I.G. Husnutdinov, L.G. Husnutdinov, D.R. Usmanov, Electromagneticacoustic method of assessing the technical condition of energy equipment, Industrial energy, 12, 8-13 (2016)

15. Patent RU No. 169803 Device for contactless control of the strained-female-deformed state and the level of damage to metal structures / Husnutdinov I.G., Bashirov M.G., Bashirov E.M., Prakhov I.V. - Published 03.04.2017. The ballot. No 10.

16. Certificate of state registration of the program for computer No. 2018617490 Assessment of the tensedeformed state and damage of metallic-acoustic structures / Bashirov M.G., Bashirova E.M., Bashirov R.A., Husnutdinova IG - Registered 07.05.2018 